\title{
STRICT LOWER SUBDIFFERENTIABILITY AND APPLICATIONS
}

\author{
H. XU ${ }^{1}$, A. M. RUBINOV ${ }^{1}$ and B. M. GLOVER ${ }^{1}$
}

(Received 6 January 1997; revised 3 November 1997)

\begin{abstract}
We investigate the strict lower subdifferentiability of a real-valued function on a closed convex subset of $\mathbb{R}^{n}$. Relations between the strict lower subdifferential, lower subdifferential, and the usual convex subdifferential are established. Furthermore, we present necessary and sufficient optimality conditions for a class of quasiconvex minimization problems in terms of lower and strict lower subdifferentials. Finally, a descent direction method is proposed and global convergence results of the consequent algorithm are obtained.
\end{abstract}

\section{Introduction}

In this paper we investigate the concept of strict lower subdifferentiability of functions and its application in mathematical programming. Strictly lower subdifferentiable functions were defined by Plastria [9] as a sub-class of lower subdifferentiable functions. There has already been considerable investigation of lower subdifferentiability and its applications, and the most important result is a relation between lower subdifferentiability and quasiconvexity. In particular, a lower subdifferentiable function can be shown to be quasiconvex and lower semicontinuous. More recently, Martinez-Legaz [5] proved that lower subdifferentiability of a function can be regarded as a special case of abstract convexity (see [7]). Further, relations to the tangential of Crouzeix [1] and the generalized subdifferential of Greenberg and Pierskalla [3] have been established (see [5]). Another remarkable result is that the classic cutting plane algorithm was extended to solve quasiconvex programming problems by using the lower subdifferential. (For details, see [9].) In contrast, there has been limited further discussion on the relationship between strict lower subdifferentiability and quasiconvexity.

Here we discuss specific characteristics of strictly lower subdifferentiable functions, their relationship to convexity, quasiconvexity, lower subdifferentiability and

\footnotetext{
'School of Information Technology and Mathematical Sciences, University of Ballarat, Victoria, Australia.

(C) Australian Mathematical Society, 1999, Serial-fee code 0334-2700/99
} 
other related properties. We show that the strict lower subdifferential coincides with the lower subdifferential at every point which is not a global minimizer and that a strictly lower subdifferentiable function defined on a closed convex set is essentially quasiconvex and under Lipschitz continuity assumptions the converse is true. We also show that under some additional assumptions the strict lower subdifferential of a strictly quasiconvex function at a given point coincides with the usual 'convex subdifferential' of a specially related convex function. Consequently we can apply the powerful methods of convex analysis to the study of the strict lower subdifferential. We study constrained and unconstrained minimization problems involving strictly lower subdifferentiable functions. In particular, we present a necessary and sufficient optimality condition for a class of essentially quasiconvex programming problems in terms of the strict lower subdifferential. Finally, we propose a descent direction method for minimizing a class of boundedly strictly lower subdifferentiable functions.

This paper is structured as follows. In Section 2 strictly lower subdifferentiable functions are introduced and their basic properties are outlined. Section 3 presents results on the structure of the strict lower subdifferential at extreme points of level sets of the given function. In Section 4 we present optimality conditions for minimization problems involving strictly lower subdifferentiable functions. Finally in Section 5 we discuss a descent direction method for a class of unconstrained quasiconvex minimization problems.

This paper is dedicated to Bruce Craven and Bert Mond on the occasion of their retirement. It is a contribution to nonsmooth analysis and mathematical programming, two areas in which Bruce Craven and Bert Mond have made substantial and deep contributions over several decades.

\section{Strict lower subdifferentiability - basic properties}

In this paper we consider a lower semicontinuous function $f: \mathbb{R}^{n} \rightarrow \mathbb{R}_{+\infty}$ with a closed convex domain denoted dom $f=\left\{x \in \mathbb{R}^{n}: f(x)<+\infty\right\}$, where $\mathbb{R}_{+\infty}=$ $\mathbb{R} \bigcup\{+\infty\}$.

Throughout this paper we shall use the following notation: $[x, y]$ will represent the inner product of vectors $x, y$ of $\mathbb{B}^{n}$ and $\|\cdot\|$ will denote the Euclidean norm. Let $\operatorname{cl} \Omega$, bd $\Omega$, and int $\Omega$ denote the closure, boundary, and interior of $\Omega$ respectively. Likewise, we use epi $f, \operatorname{co} f$, and $\mathrm{cl} f$ to denote the epigraph, convex hull, and closure of a function $f$ (see for example [11]). Let cone $\Omega=\{\lambda x: \lambda \geq 0, x \in \Omega\}$ denote the cone generated by $\Omega$.

Let $S_{f}(y)=\left\{x \in \mathbb{R}^{n}: f(x) \leq f(y)\right\}$ and $T_{f}(y)=\left\{x \in \mathbb{R}^{n}: f(x)<f(y)\right\}$. Let $D_{f}^{*}$ denote the set of global minimizers of $f$. 
A function $f$ is said to be lower subdifferentiable at a point $y$ if

$$
\left.\partial^{-} f(y)=\left\{v \in \mathbb{R}^{n}:\left(\forall x \in T_{f}(y)\right)[v, x-y] \leq f(x)-f(y)\right\} \neq \emptyset\right] .
$$

If $y \in D_{f}^{*}$, then $T_{f}(y)=\emptyset$. In this case $\partial^{-} f(y)=\mathbb{R}^{n}$. The set $\partial^{-} f(y)$ is called the lower subdifferential of the function $f$ at the point $y$. A function $f$ is called lower subdifferentiable if $\partial^{-} f(y) \neq \emptyset$ for all $y \in \operatorname{dom} f$.

A function $f$ is said to be strictly lower subdifferentiable at a point $y$ if

$$
\left.\partial_{s}^{-} f(y)=\left\{v \in \mathbb{R}^{n}:\left(\forall x \in S_{f}(y)\right)[v, x-y] \leq f(x)-f(y)\right\} \neq \emptyset\right\} .
$$

The set $\partial_{s}^{-} f(y)$ is called the strict lower subdifferential of the function $f$ at the point $y$. A function $f$ is called strictly lower subdifferentiable if $\partial_{s}^{-} f(y) \neq \emptyset$ for all $y \in \operatorname{dom} f$.

The notion of strict lower subdifferentiability and lower subdifferentiability were introduced by Plastria [9]. Clearly $\partial_{s}^{-} f(y)$ is a closed convex set. We now look into the structure of $\partial_{s}^{-} f(y)$.

Let $\Omega$ be a convex set with $y \in \Omega$. Denote the recession cone of $\Omega$ by re $\Omega=\{v$ : $\left.\left(\forall v^{\prime} \in \Omega, \lambda \geq 0\right) v^{\prime}+\lambda v \in \Omega\right\}$, and the normal cone of $\Omega$ at $y$ by $N(y, \Omega)=\{v:$ $(\forall x \in \Omega)[v, x-y] \leq 0\}$. We have the following easily established results.

PROPOSITION 2.1. If $\partial_{s}^{-} f(y)$ is nonempty, then $\partial_{s}^{-} f(y) \subseteq N\left(y, S_{f}(y)\right)=$ re $\partial_{s}^{-} f(y)$. Furthermore if $S_{f}(y)$ is convex and $y \in$ bd $S_{f}(y)$, then $\partial_{s}^{-} f(y) \neq \emptyset$.

PROPOSITION 2.2. The following hold:

(a) $0 \in \partial_{s}^{-} f(y)$ if and only if $y \in D_{f}^{*}$;

(b) the set $D_{f}^{*}$ is a singleton if and only if $\partial_{s}^{-} f(y)=\mathbb{R}^{n}$;

(c) if $y \in$ int $S_{f}(y)$ and $\partial_{s}^{-} f(y) \neq \emptyset$, then $\partial_{s}^{-} f(y)=\{0\}$.

Next, we shall compare $\partial_{s}^{-} f(y)$ and $\partial_{-} f(y)$. Obviously $\partial_{s}^{-} f(y) \subseteq \partial^{-} f(y)$, but the reverse inclusion does not necessarily hold.

PROPOSITION 2.3. Let $y \in \operatorname{dom} f$. If $\mathrm{cl} T_{f}(y)=S_{f}(y)$, then $\partial_{s}^{-} f(y)=\partial^{-} f(y)$.

Note that lower subdifferentiability implies quasiconvexity and lower semicontinuity [9]. Using Proposition 2.2, we can prove that the condition cl $T_{f}(y)=S_{f}(y)$ can be replaced by the continuity of $f$.

PROPOSITION 2.4. Suppose that int $\operatorname{dom} f \neq \emptyset$. If $f$ is continuous on $\operatorname{dom} f$ and strictly lower subdifferentiable, then $\partial_{s}^{-} f(y)=\partial^{-} f(y)$ for all $y \notin D_{f}^{*}$. 
In the following we will investigate the relationship between strict lower subdifferentiability and essential quasiconvexity. Recall that a quasiconvex function $f$ is called essentially quasiconvex if each local minimizer of $f$ is global. It is not difficult to show that every local minimizer of a strictly lower subdifferentiable function with respect to a closed convex set is a global minimizer. With this, we can obtain the following result.

PROPOSITION 2.5. Iff is strictly lower subdifferentiable on $\operatorname{dom} f$, then it is essentially quasiconvex.

The converse to Proposition 2.5 does not necessarily hold, for example, consider the function

$$
f(x)= \begin{cases}0 & x=0 \\ -x \ln (-x) & x \in[-1,0) \\ +\infty & \text { otherwise }\end{cases}
$$

However under additional assumptions we have the following result.

PROPOSITION 2.6. Suppose that int $\operatorname{dom} f$ is non-empty. If $f$ is Lipschitz and essentially quasiconvex on $\operatorname{dom} f$, then $f$ is strictly lower subdifferentiable on $\operatorname{dom} f$.

As we have shown, a strictly lower subdifferentiable function $f$ is essentially quasiconvex. There are various definitions of generalized subdifferential for quasiconvex functions (see, for example, [8] and the references therein). It is interesting to compare strict lower subdifferentiability with some of these definitions. Here we consider the following generalized subdifferential:

$$
\partial_{s}^{*} f(y)=\{v:[v, x]>[v, y] \Rightarrow f(x)>f(y)\} .
$$

PROPOSITION 2.7. Let $f$ be strictly lower subdifferentiable. Then $\partial_{s}^{-} f(y) \subseteq \partial_{s}^{*} f(y)$, for $y \in \operatorname{dom} f$.

A relationship between the lower subdifferential and the generalized subdifferential of Greenberg and Pierskalla [3] was established by Martinez-Legaz [5].

If $f$ is a convex function we can compare $\partial_{s}^{-} f(y)$ and $\partial f(y)$ which is the usual convex subdifferential, see Rockafellar [11]. The proof of the following is straightforward.

PROPOSITION 2.8. Assume that $f$ is convex and let $y \in \operatorname{ridom} f$ where $\operatorname{ridom} f$ is the relative interior of $\operatorname{dom} f$. Then

$$
\partial_{s}^{-} f(y)=\partial f(y)+N\left(y, S_{f}(y)\right) .
$$


We now analyse the set valued mapping $x \longmapsto \partial_{s}^{-} f(x)$.

PROPOSITION 2.9. Suppose that int $\operatorname{dom} f \neq \emptyset$ and $f$ is continuous on $\operatorname{dom} f$ and strictly lower subdifferentiable. Then the mapping $x \mapsto \partial_{s}^{-} f(x)$ is closed on dom $f$.

Proof. Let $x_{k} \rightarrow x, v_{k} \rightarrow v$ with $v_{k} \in \partial_{s}^{-} f\left(x_{k}\right)$. We must verify that $v \in \partial_{s}^{-} f(x)$. First assume $x \notin D_{f}^{*}$. Then $x_{k} \notin D_{f}^{*}$ for sufficiently large $k$, say for $k>K$. Applying Proposition 2.4 we conclude that $\partial_{s}^{-} f(x)=\partial^{-} f(x)$ and $\partial_{s}^{-} f\left(x_{k}\right)=\partial^{-} f\left(x_{k}\right)$ for $k>K$. Let $y \in T_{f}(x)$ and $\varepsilon=\frac{1}{2}(f(x)-f(y))$. Since $f$ is continuous, for sufficiently large $k>K$, we have $f\left(x_{k}\right)>f(x)-\varepsilon>f(y)$, and so $y \in T_{f}\left(x_{k}\right)$. Since $v_{k} \in \partial_{s}^{-} f\left(x_{k}\right)=\partial^{-} f\left(x_{k}\right)$, we have $\left[v_{k}, y-x_{k}\right] \leq f(y)-f\left(x_{k}\right)$. Thus $[v, y-x] \leq f(y)-f(x)$ and $v \in \partial^{-} f(x)=\partial_{s}^{-} f(x)$. Secondly, suppose $x \in D_{f}^{*}$. Then $\partial_{s}^{-} f(x)=\mathbb{R}^{n}$ and therefore $v \in \partial_{s}^{-} f(x)$.

\section{The strict lower subdifferential at level set extreme points}

Let $g: \mathbb{R}^{n} \rightarrow \mathbb{R}_{+\infty}$ be a convex function. If dom $g=S_{g}(y)$, then $\partial_{s}^{-} f(y)=\partial f(y)$. We will use this relationship to study strict lower subdifferentiability.

Throughout this section let $f$ be a quasiconvex lower semicontinuous function and let $y \in \operatorname{dom} f$. Then $S_{f}(y)$ is closed and convex. Assume that $\partial_{s}^{-} f(y)$ is not empty. Set

$$
f_{y}(x)= \begin{cases}f(x) & x \in S_{f}(y), \\ +\infty & x \notin S_{f}(y) .\end{cases}
$$

Then $\partial_{s}^{-} f(y)=\partial_{s}^{-} f_{y}(y)$. We shall use the function $f_{y}$ to study $\partial_{s}^{-} f(y)$. Let co $f$ be the convex hull of the function $f$ (that is co(epi $f$ ) $=\operatorname{epi}(\operatorname{co} f)$ ) and let $(\operatorname{clco} f)(x)=\liminf _{x^{\prime} \rightarrow x}(\operatorname{co} f)\left(x^{\prime}\right)$ be the closure of $f$. It is well known (see [11]) that $\operatorname{cl} \operatorname{co} f=f^{* *}$ where $f^{*}$ denotes the Fenchel conjugate function to $f$, that is, $f^{*}(v)=\sup _{x \in \mathbb{R}^{n}}\{[v, x]-f(x)\}$, and $f^{* *}=\left(f^{*}\right)^{*}$. Thus (see [11]) $(\operatorname{clco} f)(x)=$ $\sup _{(v, c) \in s(f)}([v, x]+c)$, where

$$
\mathrm{s}(f)=\left\{(v, c) \in \mathbb{R}^{n} \times \mathbb{R}:\left(\forall x \in \mathbb{R}^{n}\right)[v, x]-c \leq f(x)\right\}=\operatorname{epi} f^{*} .
$$

THEOREM 3.1. If $y$ is an extreme point of $S_{f}(y)$, then

$$
\partial_{s}^{-} f(y)=\partial\left(\operatorname{co} f_{y}\right)(y)
$$

We need the following lemmas in the proof of this theorem. 
LEMMA 3.1. If $\operatorname{dom} f^{* *}$ is bounded, then $f^{* *}=\operatorname{co} f$.

This result follows directly from [4, Proposition 1.5.4]. Using the definition of a convex hull of a function and the definition of an extreme point, we can also obtain the following result.

LEMMA 3.2. If the conditions of Theorem 3.1 are satisfied, then $\operatorname{cl} \operatorname{co} f_{y}=\operatorname{co} f_{y}$ and $\left(\operatorname{cof} f_{y}\right)(y)=f(y)$.

PROOF OF THE THEOREM. First we will prove the inclusion

$$
\partial_{s}^{-} f(y) \subseteq \partial f_{y}(y)
$$

Take the set

$$
\mathrm{s}\left(f_{y}\right)=\left\{(v, c):\left(\forall x \in S_{f}(y)\right)[v, x]-c \leq f(x)\right\} .
$$

Since the lower semicontinuous function $f$ is bounded below on the compact set $S_{f}(y)$ it follows that $s\left(f_{y}\right)$ is nonempty. Applying Lemma 3.2 we conclude that

$$
\left(\operatorname{co} f_{y}\right)(x)=\left(\operatorname{clco} f_{y}\right)(x)=\sup \left\{[v, x]-c:(v, c) \in \mathrm{s}\left(f_{y}\right)\right\}, \quad \text { for all } x \in S_{f}(y) .
$$

For $v \in \partial_{s}^{-} f(y)$ we have $[v, x-y] \leq f(x)-f(y)$ for all $x \in S_{f}(y)$. Let $c=$ $[v, y]-f(y)$. Then for all $x \in S_{f}(y),[v, x]-c \leq f(x)$ and

$$
\begin{aligned}
\left(\operatorname{co} f_{y}\right)(x) & =\left(\operatorname{clco} f_{y}\right)(x)=\sup _{\left(v^{\prime}, c^{\prime}\right) \in s(f)}\left(\left[v^{\prime}, x\right]-c^{\prime}\right) \geq[v, x]-c \\
& =[v, x]-[v, y]+f(y)=[v, x]-[v, y]+\left(\operatorname{co} f_{y}\right)(y) .
\end{aligned}
$$

Since dom $f_{y}=S_{f}(y)$ it follows that $v \in \partial f_{y}(y)$. Thus (1) has been proved. Now we prove the reverse inclusion. Since $\operatorname{co} f_{y}(x) \leq f_{y}(x)=f(x)$ for all $x \in S_{f}(y)$ and co $f_{y}(y)=f_{y}(y)=f(y)$ we have, for $v \in \partial f_{y}(y)$ and any $x \in S_{f}(y)$,

$$
[v, x-y] \leq \operatorname{co} f_{y}(x)-\operatorname{co} f_{y}(y) \leq f(x)-f(y) .
$$

Thus $v \in \partial_{s}^{-} f(y)$.

COROLlaRY 3.1. Assume that $\operatorname{dom} f$ is bounded. If $f$ is strictly quasiconvex in the following sense:

$$
(\forall x, y \in \operatorname{dom} f, x \neq y, \forall \alpha \in(0,1)) \quad f(\alpha x+(1-\alpha) y)<\max \{f(x), f(y)\},
$$

then $\partial_{s}^{-} f(y)=\partial\left(\operatorname{co} f_{y}\right)(y)$ for all $y \in \operatorname{dom} f$. 
Theorem 3.1 allows us to apply the methods of convex analysis for studying the strict lower subdifferential at a point $y$ which is an extreme point of the set $S_{f}(y)$.

PROPOSITION 3.1. Assume that the conditions of Theorem 3.1 hold. Then the following are equivalent:

(a) $v \in \partial_{s}^{-} f(y)$;

(b) The function $x \mapsto[v, x]-f(x)$ achieves its supremum over the set $S_{f}(y)$ at the point $y$;

(c) $f(y)+\left(f_{y}\right)^{*}(v)=[v, y]$;

(d) $f(y)+\left(f_{y}\right)^{*}(v) \leq[v, y]$;

(e) $y \in \partial\left(f_{y}\right)^{*}(v)$.

PROOF. The results follow directly from Theorem 3.1 and [11, Theorem 23.5].

We can exploit Theorem 3.1 in the study of the strict lower $\varepsilon$-subdifferential. For $\epsilon>0$, the set

$$
\partial_{s, \epsilon}^{-} f(y)=\left\{v:\left(\forall x \in S_{f}(y)\right)[v, x-y]-\varepsilon \leq f(x)-f(y)\right\}
$$

is called the strict lower $\varepsilon$-subdifferential of $f$ at $y$.

PROPOSITION 3.2. Suppose that the conditions of Theorem 3.1 hold for the function $f$ and the point $y$. Then, for $\varepsilon>0$, we have

$$
\partial_{s, \epsilon}^{-} f(y)=\partial_{\varepsilon}\left(\operatorname{co} f_{y}\right)(y),
$$

where $\partial_{\varepsilon}\left(\operatorname{co} f_{y}\right)(y)$ is the usual $\varepsilon$-subdifferential of the convex function $\operatorname{co} f_{y}$ at the point $y$.

\section{Optimality conditions}

In this section we consider the following minimization problem:

$$
\begin{aligned}
& \text { (P) } \min f(x) \\
& \text { subject to } x \in C,
\end{aligned}
$$

where $\operatorname{dom} f=\mathbb{R}^{n}, C$ is a closed convex set with int $C \neq \emptyset$. Let $\operatorname{ker}(f)=\{x$ : $f(x)=f(0)\}$.

ASSUMPTION 4.1. Assume $f$ satisfies the following: $f(0)=\inf \left\{f(x): x \in \mathbb{R}^{n}\right\}$, and $\operatorname{ker}(f) \cap C=\emptyset$. 
This assumption is reasonable in practice (see [12] for a discussion). Note that Thach and Kojima [12] presented a necessary and sufficient optimality condition for $(\mathrm{P})$ in terms of the generalized subdifferential under Assumption 4.1 and essential quasiconvexity. In what follows, we shall propose a necessary and sufficient optimality condition for $(\mathrm{P})$ in terms of (strict) lower subdifferentials under similar assumptions.

THEOREM 4.1. Let $f$ be locally Lipschitz on $\mathbb{R}^{n}$, lower subdifferentiable at every point in $C$, and essentially quasiconvex on $C$. Assume that Assumption 4.1 holds, $x^{*} \in C$, and $T_{f}\left(x^{*}\right)$ is bounded. Then $x^{*}$ is optimal for $(P)$ if and only if

$$
0 \in \partial^{-} f\left(x^{*}\right)+N\left(x^{*}, C\right) .
$$

PRoof. Suppose that $x^{*}$ satisfies (2). Then there exists $v \in \partial^{-} f\left(x^{*}\right)$ such that $-v \in N\left(x^{*}, C\right)$. By definition, for each $x \in T_{f}\left(x^{*}\right), 0>f(x)-f\left(x^{*}\right) \geq\left[v, x-x^{*}\right]$. Equivalently if there exists a point $x$ such that $\left[v, x-x^{*}\right] \geq 0$, then $x \notin T_{f}\left(x^{*}\right)$. On the other hand, for every $x \in C,\left[v, x-x^{*}\right] \geq 0$. Thus, $x \notin T_{f}\left(x^{*}\right)$, for every $x \in C$, which proves that $x^{*}$ is a global minimizer of $f$ over $C$.

Conversely, if $x^{*}$ is a global minimizer of $(\mathrm{P})$, then $x^{*} \in$ bd $C$, otherwise $x^{*} \in$ int $C$ is the global minimizer of $f$ which contradicts our assumption.

Clearly $C \cap T_{f}\left(x^{*}\right)=\emptyset$. Since $f$ is continuous it follows that $T_{f}\left(x^{*}\right)$ is open. By the separation theorem, there exists a $v \neq 0$, with $\|v\|=1$ such that, for each $x \in T_{f}\left(x^{*}\right),\left[v, x-x^{*}\right]<0$ and, for every $x^{\prime} \in C,\left[v, x^{\prime}-x^{*}\right] \geq 0$. Let $P$ denote the plane $\left\{x:\left[v, x-x^{*}\right]=0\right\}, \bar{x}$ denote the orthogonal projection of $x \in T_{f}\left(x^{*}\right)$ onto $P$. Clearly $\bar{x} \notin T_{f}\left(x^{*}\right)$. Thus $f(x)-f\left(x^{*}\right) \geq f(x)-f(\bar{x})$. Since $T_{f}\left(x^{*}\right)$ is bounded, then the projection of $T_{f}\left(x^{*}\right)$ on $P$ is bounded. By assumption there exists a positive constant $L$ such that $f(x)-f(\bar{x}) \geq-L\|x-\bar{x}\|$. Since $\|x-\bar{x}\|=\left[v, x^{*}-x\right]$, then $f(x)-f\left(x^{*}\right) \geq\left[L v, x-x^{*}\right]$. Note that $x$ is arbitrarily taken from $T_{f}\left(x^{*}\right)$. We conclude that $L v \in \partial^{-} f\left(x^{*}\right)$. Note also that $\left[L v, x^{\prime}-x^{*}\right] \geq 0$ for all $x^{\prime} \in C$. This proves (2) as required.

By applying Proposition 2.4, Proposition 2.5 and the above theorem, we have the following result.

COROLLARY 4.1. Let $f$ be locally Lipschitz on $\mathbb{R}^{n}$, and strictly lower subdifferentiable on $\mathbb{R}^{n}$. Assume that Assumption 4.1 holds, $x^{*} \in C$, and $T_{f}\left(x^{*}\right)$ is bounded. Then $x^{*}$ is optimal for $(P)$ if and only if $0 \in \partial_{s}^{-} f\left(x^{*}\right)+N\left(x^{*}, C\right)$.

When $C$ is represented by a finite number of inequalities, we have the following. 
THEOREM 4.2. Let $g_{i}$ be locally Lipschitz on $\mathbb{R}^{n}, C_{i}=\left\{x \in R^{n}: g_{i}(x) \leq 0\right\},(i=$ $1, \ldots, m), C=\bigcap_{i=1}^{m} C_{i}$, and int $C \neq \emptyset$. Assume that, for $i=1, \ldots, m, C_{i}$ is convex, and $g_{i}$ is lower subdifferentiable at every point in $C_{i}$. Assume also that $f$ is locally Lipschitz on $\mathbb{B}^{n}$, lower subdifferentiable at every point in $C$, and essentially quasiconvex on $C$. Let $x^{*} \in C$. Then $x^{*}$ is optimal for $(P)$ if and only if

$$
0 \in \partial^{-} f\left(x^{*}\right)+\sum_{i=1}^{m} \text { cone } \partial^{-} g_{i}\left(x^{*}\right) \text {. }
$$

PROOF. By Theorem 4.1, it suffices to prove that $\sum_{i=1}^{m}$ cone $\partial^{-} g_{i}\left(x^{*}\right)=N\left(x^{*}, C\right)$. Let $K(x, C)$ denote the tangent cone of $C$ at $x$. Since $C_{i}$ is convex, it is well-known that $K\left(x^{*}, C\right)=\bigcap_{i=1}^{m} K\left(x^{*}, C_{i}\right)$, and $N\left(x^{*}, C\right)=-\left(K\left(x^{*}, C\right)\right)^{*}$, where $\left(K\left(x^{*}, C\right)\right)^{*}$ denotes the conjugate cone of $K\left(x^{*}, C\right)$. Also

$$
\begin{aligned}
N\left(x^{*}, C\right) & =-\left(K\left(x^{*}, C\right)\right)^{*}=-\left(\bigcap_{i=1}^{m} K\left(x^{*}, C_{i}\right)\right)^{*} \\
& =-\operatorname{cl}\left[\sum_{i=1}^{m}\left(K\left(x^{*}, C_{i}\right)\right)^{*}\right]=\mathrm{cl}\left[\sum_{i=1}^{m} N\left(x^{*}, C_{i}\right)\right] .
\end{aligned}
$$

By [5, Proposition 4.17], $N\left(x^{*}, C_{i}\right)=$ cone $\partial^{-} g_{i}\left(x^{*}\right)$. Since int $C \neq \emptyset$, it follows that int $K\left(x^{*}, C\right) \neq \emptyset$ and therefore the cone $N\left(x^{*}, C\right)=-\left(K\left(x^{*}, C\right)\right)^{*}$ has a compact base. It is well-known that the sum of closed cones contained in a cone with a compact base is also closed. The proof is complete.

\section{Descent direction methods}

In this section, we shall discuss numerical methods for minimizing a boundedly strictly lower subdifferentiable function over a closed convex set $C \subset \mathbb{R}^{n}$. There have been some specific numerical methods developed for solving quasiconvex minimization problems. The most noteworthy ones are Gromicho's ellipsoid method and Plastria's cutting plane method.

In this section, we shall discuss an alternative approach by introducing a descent direction method for solving a special class of unconstrained quasiconvex minimization problems.

A function $f$ is said to be boundedly strictly lower subdifferentiable at a point $y \in \operatorname{dom} f$ if there exists a positive constant $M$ such that the following set is nonempty

$$
\partial_{M}^{-} f(y)=\partial_{s}^{-} f(y) \bigcap\left\{v \in \mathbb{R}^{n}:\|v\| \leq M\right\} .
$$

From the definition, it is clear that $\partial_{M}^{-} f(y)$ is a closed convex set. 
THEOREM 5.1. Assume that $f$ is continuous on $\operatorname{dom} f$ and boundedly strictly lower subdifferentiable at every point of a compact convex set $C \subseteq \operatorname{dom} f$. Then $\partial_{M}^{-} f(\cdot)$ is upper semicontinuous on $C$.

PROOF. It is well-known that a closed set-valued mapping with compact graph is upper semicontinuous, see, for example, [6]. Hence it suffices to show that $\partial_{M}^{-} f(\cdot)$ is closed. Let $x_{k} \in C$ and $x_{k} \rightarrow x, v_{k} \in \partial_{M}^{-} f\left(x_{k}\right)$, and $v_{k} \rightarrow v$. We need to prove that $v \in$ $\partial_{M}^{-} f(x)$. By definition, $\left\|v_{k}\right\| \leq M$, thus $\|v\| \leq M$. Since $\partial_{M}^{-} f\left(x_{k}\right) \subset \partial_{s}^{-} f\left(x_{k}\right)$, and $\partial_{s}^{-} f(\cdot)$ is closed (by Proposition 2.9), we have $v \in \partial_{s}^{-} f(x)$. Therefore $v \in \partial_{M}^{-} f(x)$. The proof is complete.

Using Theorem 5.1, we can easily obtain the following.

LEMMA 5.1. Assume that $f$ is boundedly strictly lower subdifferentiable at every point of a compact set $C \subseteq \operatorname{dom} f$, with $x \in$ int $C, d \in \mathbb{R}^{n}$, and $d \neq 0$. If $d$ is not a descent direction, then there exists $v \in \partial_{M}^{-} f(x)$, such that $[d, v] \geq 0$.

THEOREM 5.2. Assume that $f$ is boundedly strictly lower subdifferentiable at every point of a compact set $C \subseteq \operatorname{dom} f$, and let $x \in \operatorname{int} C$. If $0 \notin \partial_{M}^{-} f(x)$, then there exists $a v \in \partial_{M}^{-} f(x)$ such that $-v$ is a descent direction of $f$ at $x$.

ProOF. For the sake of contradiction assume that, for every $v \in \partial_{M} f(x),-v$ is not a descent direction. Then, by Lemma 5.1, there exists $v^{*} \in \partial_{M}^{-} f(x)$, such that $\left[v, v^{*}\right] \leq 0$. Let $a(v)=\left\{v^{*} \in \partial_{M}^{-} f(x):\left[v, v^{*}\right] \leq 0\right\}$. Then for all $v \in \partial_{M}^{-} f(x)$, $a(v) \neq \emptyset, a(v) \subset \partial_{M}^{-} f(x)$, and $a(v)$ is convex. Further $a(\cdot)$ is closed. By the Kakutani fixed point theorem, there exists $v \in \partial_{M}^{-} f(x)$ such that $[v, v]=\|v\|^{2} \leq 0$, thus $0 \in \partial_{M}^{-} f(x)$, a contradiction.

LEMMA 5.2 ([2]). Let $C \subseteq \operatorname{dom} f$ be a closed convex set in $\mathbb{R}^{n}$ with $0 \notin C$ and $d^{*}=\arg \min \{\|d\|: d \in C\}$. Then $\left[d, d^{*}\right]>0$ for all $d \in C$.

Now let

$$
h=-\arg \min \left\{\|v\|: v \in \partial_{M}^{-} f(x)\right\} .
$$

We have the following theorem.

THEOREM 5.3. Assume that $f$ is Lipschitz and strictly lower subdifferentiable at every point of a compact set $C \subseteq \operatorname{dom} f$ and let $x \in \operatorname{int} C$. If $0 \notin \partial_{M}^{-} f(x)$, then $h$, defined by (3), is a descent direction. 
PROOF. For the sake of a contradiction, assume $h$ is not a descent direction. Then, by Lemma 5.1, there exists $v^{*} \in \partial_{M}^{-} f(x)$ such that $\left[h, v^{*}\right] \geq 0$. Also, since $\partial_{M}^{-} f(x)$ is convex and $0 \notin \partial_{M}^{-} f(x)$, by Lemma 5.2, $\left[h, v^{*}\right]<0$, which leads to the desired contradiction.

By Theorem 5.3, we can find a descent direction for a boundedly strictly lower subdifferentiable function $f$ at the interior of a compact set when $0 \notin \partial_{M}^{-} f(x)$. The process is summarised in the following algorithm.

ALGORITHM 5.1 (Finding a Descent Direction). Step 1. Let $\mathscr{A}_{0} \subseteq \partial_{M}^{-} f(x)$ be a convex set, set $k:=0$.

Step 2. Compute $h_{k}=-\arg \min \left\{\|h\|: h \in \mathscr{A}_{k}\right\}$, if $h_{k}$ is a descent direction, then stop.

Step 3. Find $a_{k} \in \partial_{M}^{-} f(x)$, such that $\left[h_{k}, a_{k}\right]>0$, and set $\mathscr{A}_{k+1}=\operatorname{co}\left(\mathscr{A}_{k} \bigcup\left\{a_{k}\right\}\right)$, $k:=k+1$, go to Step 2 .

PROPOSITION 5.1. Let $\left\{h_{k}\right\}$ be generated by Algorithm 5.1. Suppose that $0 \notin \partial_{M}^{-} f(x)$. Then

(a) $\left\|h_{k+1}\right\|<\left\|h_{k}\right\|$;

(b) the process must terminate in a finite number of steps.

PROOF. (a) By the definition of $h_{k}$ we have $\left[a, h_{k}\right]<0$ for all $a \in \mathscr{A}_{k}$. Since $\left[h_{k}, a_{k}\right]>0$, we have $a_{k} \notin \mathscr{A}_{k}$. Thus $\mathscr{A}_{k} \subset \mathscr{A}_{k+1} \subseteq \partial_{M}^{-} f(x)$. The last inclusion is due to the convexity and closedness of $\partial_{M}^{-} f(x)$. Let $\lambda=\left[h_{k}, a_{k}+h_{k}\right] /\left\|a_{k}+h_{k}\right\|^{2}$. Then $\lambda \in(0,1), h_{\lambda}=-h_{k}+\lambda\left(a_{k}+h_{k}\right) \in \mathscr{A}_{k+1}$, and by a simple calculation, $\left\|h_{\lambda}\right\|^{2}=\left\|h_{k}\right\|^{2}-\lambda^{2}\left\|a_{k}+h_{k}\right\|^{2}<\left\|h_{k}\right\|^{2}$. Thus $\left\|h_{k+1}\right\|^{2}<\left\|h_{k}\right\|^{2}$.

(b) For the sake of a contradiction, assume that the process is infinite. Since $a_{j} \in \mathscr{A}_{k}$, for $j=1, \ldots, k-1$, and $-h_{k} \in \mathscr{A}_{k}$, then $\left[a_{j},-h_{k}\right] \geq\left\|h_{k}\right\|^{2}$. Note that $\left[h_{k}, a_{k}\right]>0$. Thus $\left[h_{k}, a_{k}-a_{j}\right] \geq\left\|-h_{k}\right\|^{2}$. Note also that $\left[h_{k+1}, h_{k}\right]=$ $\left[-h_{k+1},-h_{k}\right] \geq\left\|-h_{k+1}\right\|^{2}>0$ and $\left\|a_{k}\right\|$ is bounded by $M$. By [4, Lemma 2.1.1], $h_{k} \rightarrow 0$. Note that $-h_{k} \in \partial_{M}^{-} f(x)$ and $\partial_{M}^{-} f(x)$ is closed. This leads to a contraction.

The result above allows the calculation of a descent direction for a boundedly strictly lower subdifferentiable function $f$ at a point $x$ where $0 \notin \partial_{M}^{-} f(x)$. Indeed, a descent direction can be calculated using the Clarke subdifferential $\partial_{c} f(x)$. More precisely, if $0 \notin \partial_{c} f(x)$, then a descent direction can be found. In the past few decades several descent direction methods using the Clarke subdifferential have been developed, such as the $\epsilon$-subgradient method and its variations (see [10]). These results have been shown to be both robust and efficient. It is reasonable therefore to propose an algorithm which incorporates the main features of these well-known 
algorithms with a specific technique for dealing with Clarke stationary points, (that is, points at which $0 \in \partial_{c} f(x)$ ).

We now describe such a hybrid algorithm.

AlgorithM 5.2 (A Descent Direction Method). Step 1. Let $x_{0} \in \operatorname{dom} f$, set $k=0$. Step 2. Let $y_{0}=x_{k}$. Take $y_{0}$ as a starting point and use the $\epsilon$-subgradient algorithm to minimize $(P)$ until the algorithm terminates at some point $y$ such that $0 \in \partial_{c} f(y)$, set $x_{k}=y$;

Step 3. Compute $h_{k}$ with Algorithm 5.1. If $h_{k}=0$, then stop;

Step 4. Compute stepsize: $t_{k}=\arg \min _{\imath>0}\left\{f \cdot\left(x_{k}+t h_{k}\right)\right\}$. Set $x_{k+1}=x_{k}+t_{k} h_{k}, k:=$ $k+1$, go to Step 2 .

THEOREM 5.4. Assume that $f$ is continuous and boundedly strictly lower subdifferentiable at every point of any compact convex subset of $\operatorname{dom} f$ and let $\left\{x_{k}\right\}$ be generated by Algorithm 5.2 with $x_{0} \in \operatorname{int} \operatorname{dom} f$. Suppose that $S_{f}\left(x_{0}\right)$ is bounded, $0 \notin \partial_{c} f\left(x_{0}\right)$, and $f$ has no point with a neighborhood containing an infinite number of Clarke stationary points. Then the sequence $\left\{x_{k}\right\}$ generated by Algorithm 5.2 converges to the global minimizer of $f$.

PROOF. By assumption, $f\left(x_{1}\right)<f\left(x_{0}\right)$. Let $S_{0}=\left\{x \in \mathbb{R}^{n}: f(x) \leq\left[f\left(x_{1}\right)+\right.\right.$ $\left.\left.f\left(x_{0}\right)\right] / 2\right\}$. Then for $k \geq 1,\left\{x_{k}\right\} \subseteq S_{0} \subset T_{f}\left(x_{0}\right) \subset S_{f}\left(x_{0}\right)$. Thus, the sequence and its accumulation points remain in the interior of a compact set.

Assume that the algorithm terminates at some finite $k_{0}$. Note that $x_{k_{0}} \in S_{0} \subset$ $T_{f}\left(x_{0}\right) \subset S_{f}\left(x_{0}\right)$, that is, $x_{k_{0}}$ is in the interior of a compact set. Then $0 \in \partial_{M}^{-} f\left(x_{k_{0}}\right)$ and $x_{k_{0}}$ is the global minimizer.

Now assume that $x$ is an accumulation point of $\left\{x_{k}\right\}$. Then there is a $\delta>0$ such that $B(x, \delta) \subset T_{f}\left(x_{0}\right)$, where $B(x, \delta)$ denotes the $\delta$-neighborhood of $x$. If $0 \in \partial_{M}^{-} f(x)$, then $x$ is the global minimizer. Otherwise, let $\delta$ be sufficiently small so that $B(x, \delta)$ does not contain any other Clarke stationary points except $x$. That means that whenever there is an iterate $x_{k}$ which occurs in $B(x, \delta)$, the $\epsilon$-subgradient algorithm terminates at $x$. But this is impossible since $0 \notin \partial_{M}^{-} f(x)$, thus a descent direction can be found at $x$. The proof is complete.

\section{Acknowledgements}

The authors gratefully acknowledge the contribution of Dr B. Simsek to an earlier version of this paper, they are also grateful to the anonymous referee for insightful comments. Finally they wish to thank $\mathrm{Dr} \mathrm{B}$. Craven for his many helpful discussions and to Dr D. Ralph for his valuable comments. This research was partly supported by an Australian Research Council grant. 


\section{References}

[1] J. P. Crouzeix, "Contributions a l'etude des fonctions quasiconvexes", Ph. D. Thesis, Universite de Clermont-Ferrand II, Clermont-Ferrand, 1977.

[2] V. F. Demyanov and V. Malozemov, Introduction to Minimax (John Wiley and Sons, New York, 1974).

[3] H. P. Greenberg and W. P. Pierskalla, "Quasiconjugate function and surrogate duality", Cahiers du Centre d'etudes de Recherche Operationnelle 15 (1973) 437-448.

[4] J.-B. Hiriart-Urruty and C. Lemarechal, Convex Analysis and Minimization Algorithms II, Fundamentals (Springer, Berlin, 1993).

[5] J. E. Martinez-Legaz, "On lower subdifferentiable functions, trends in mathematical optimization", in Proceedings, International Conference of Irsee, (Birkhauser, Boston, MA, 1986), 197-232.

[6] H. Nikaido, Convex Structures and Economic Theory (Academic Press, New-York, 1969).

[7] D. Pallaschke and S. Rolewicz, Foundations of Mathematical Optimization (Kluwer Academic Publishers, Dordrecht, 1997).

[8] J.-P. Penot and M. Volle, "On quasiconvex duality", Mathematics of Operations Research 15 (1990) 579-625.

[9] F. Plastria, "Lower subdifferentiable functions and their minimization by cuttung planes", J. Optim. Theory Appl. 46 (1985) 37-53.

[10] E. Polak, D. Q. Mayne and Y. Wardi, "On the extension of constrained optimization algorithms from differentiable to nondifferentiable problems", SIAM J. of Control and Optimization 21 (1983) 179-203.

[11] R. T. Rockafellar, Convex Analysis (Princeton University Press, Princeton, 1972).

[12] P. T. Thach and M. A. Kojima, "A generalized convexity and variational inequality for quasi-convex minimization", SIAM J. of Optimization 6 (1996) 212-226. 